\title{
SAÚDE ÚNICA E COVID-19: REVISÃO SOBRE O POTENCIAL DOS ANIMAIS COMO RESERVATÓRIOS DO VÍRUS
}

\author{
Karen Freitas ${ }^{1}$ \\ Rutiele Silveira ${ }^{2}$ \\ Antônio Barbosa ${ }^{3}$
}

\begin{abstract}
RESUMO
A doença COVID-19, causada pelo agente SARS-COV-2, provém da mutação do vírus SARS-COV de origem animal, tornando-se relevante não somente por causar infecções em humanos como também por apresentar um possível caráter zoonótico. Com essa possibilidade, estudos buscam a comprovação da presença do agente em diversas espécies de animais domésticos e quais as similaridades entre essas e o ser humano. Revisão de literatura de diversas publicações científicas abrangendo a possibilidade de os animais serem reservatórios do agente do novo coronavírus, bem como, acentuar a importância da saúde única no controle de doenças através da pesquisa de publicações, foram realizadas até 10 de Junho de 2020. Em estudos realizados com animais de companhia, cães e gatos, testados através do exame de reação em cadeia da polimerase em tempo real (RT-PCR), que permite identificar a presença do agente infeccioso no material genético do paciente, alguns dos animais avaliados apresentaram resultado positivo para o novo coronavírus. Contudo, em relação a animais de produção, até o momento, não foram detectados animais positivos para o novo coronavírus, sugerindo que estes não são suscetíveis a esta infecção. Com esta revisão, ressalta-se que é notório o potencial zoonótico do novo coronavírus a partir de estudos já existentes, porém ainda são necessários mais estudos abordando esta temática. Ademais, evidencia-se a importância da ação da saúde única de forma multiprofissional, na qual atuam médicos, enfermeiros, médicos veterinários, entre tantos, diante de todas fontes envolvidas na disseminação de doenças, como o COVID-19.
\end{abstract}

Palavras-chave: medicina veterinária, SARS-COV-2, transmissão, zoonoses.

\section{ONE HEALTH AND COVID-19: REVIEW OF THE POTENTIAL OF ANIMALS TO BE RESERVOIRS OF THE VIRUS}

\begin{abstract}
The disease COVID-19, caused by the agent SARS-COV-2, comes from the mutation of the SARS-COV virus of animal origin, becoming relevant not only for causing infections in humans but also for having a possible zoonotic character. With this possibility, studies seek to prove the presence of the agent in several species of domestic animals and what are the similarities between these and the human being. An literature review of several scientific publications covering the possibility of animals being reservoirs of the new coronavirus agent, as well as to emphasize the importance of unique health in disease control through the search of publications made up to 10 June 2020. In studies with companion animals, dogs and cats, tested using the real-time polymerase chain reaction (RT-PCR) test, which allows the

\footnotetext{
${ }^{1}$ Universidade Federal de Pelotas

${ }^{2}$ Universidade Federal de Pelotas - Graduanda em Medicina Veterinária

${ }^{3}$ Universidade Federal de Pelotas - Doutor em Ciências pela Universidade Federal de Pelotas. Contato principal: antoniobarbosa.vet@hotmail.com
}

Freitas K, Silveira R, Barbosa A. Saúde Única e COVID-19: Revisão sobre o potencial dos animais como reservatórios do vírus. Vet. e Zootec. 2020; 27: 001-007. 
presence of the infectious agent in the genetic material of patients, some of the animals evaluated tested positive for the new coronavirus. However, in relation to production animals, so far, no positive animals have been detected for the new coronavirus, suggesting that they are not susceptible to this infection. With this review, it is emphasized that the zoonotic potential of the new coronavirus is notorious from existing studies, but further studies addressing this theme are still needed. In addition, the importance of a single health action in a multidisciplinary way is evidenced, in which doctors, nurses, veterinarians, among many, before all sources involved in the spread of diseases, such as COVID-19.

Keywords: veterinary medicine, SARS-COV-2, transmission, zoonoses.

\section{SALUD ÚNICA Y COVID-19: REVISIÓN DEL POTENCIAL DE LOS ANIMALES PARA SER RESERVORIOS DEL VIRUS}

\section{RESUMEN}

La enfermedad COVID-19, causada por el agente SARS-COV-2, proviene de la mutación del virus SARS-COV de origen animal, volviéndose relevante no solo por causar infecciones en humanos sino también por tener un posible carácter zoonótico. Con esta posibilidad, los estudios buscan demostrar la presencia del agente en varias especies de animales domésticos y cuáles son las similitudes entre estos y el ser humano. Se realizó una revisión de la literatura de varias publicaciones científicas, que cubre la posibilidad de que los animales sean reservorios del nuevo agente de coronavirus, así como enfatiza la importancia de una salud única en el control de enfermedades a través de la investigación de publicaciones realizadas hasta el 10 de junio de 2020 En estudios realizados con animales de compañía, perros y gatos, probados mediante reacción en cadena de la polimerasa en tiempo real (RT-PCR), que permite la presencia del agente infeccioso en el material genético del paciente, algunos de los animales evaluados mostró resultados positivos para el nuevo coronavirus. Sin embargo, en relación con los animales de granja, hasta el momento, no se han detectado animales positivos para el nuevo coronavirus, lo que sugiere que no son susceptibles a esta infección. Con esta revisión, se enfatiza que el potencial zoonótico del nuevo coronavirus es notorio por los estudios existentes, pero aún se necesitan más estudios que aborden este tema. Además, se evidencia la importancia de una sola acción de salud de manera multidisciplinaria, en la que los médicos, enfermeras, veterinarios trabajan, entre muchos, antes de que todas las fuentes involucradas en la propagación de enfermedades, como COVID-19.

Palabras claves: medicina veterinária, SARS-COV-2, transmisión, zoonosis.

\section{INTRODUÇÃO}

No final de 2019 na China, um grupo de pessoas que coabitavam a mesma região, apresentaram sintomatologia clínica de tosse, febre e comprometimento pulmonar, porém, não havia conhecimento da sua etiologia. A partir disso, foram realizadas diversas pesquisas para identificar o agente posteriormente denominado SARS-COV-2, da doença COVID-19, que provém da mutação do vírus SARS-COV, causador da síndrome respiratória aguda severa. Ambos são pertencentes à família dos coronavírus, os quais são responsáveis por causar outras enfermidades em humanos e animais $(1,2)$.

Estes vírus são do tipo RNA, assim, têm como característica alta taxa de mutação, podendo resultar ao aumento de virulência, adaptação a novos hospedeiros e tropismo tecidual (3). Classificados em quatro gêneros, Alphacoronavirus, Betacoronavirus, Gammacoronavirus e Deltacoronavirus, os coronavírus do gênero beta possuem aspecto zoonótico, exemplo disso

Freitas K, Silveira R, Barbosa A. Saúde Única e COVID-19: Revisão sobre o potencial dos animais como reservatórios do vírus. Vet. e Zootec. 2020; 27: 001-007. 
são os vírus MERS-COV e SARS-COV. O SARS-COV-2 também é integrante deste grupo, porém, mais pesquisas são necessárias para identificar o verdadeiro potencial zoonótico deste último (4).

Devido a esta informação, buscou-se saber qual a fonte de infecção do novo coronavírus e a partir de análises moleculares, encontraram $96 \%$ de semelhança genética com o coronavírus de morcegos (5) e 90,5\% de similaridade em isolados de pangolins resgatados do comércio ilegal, possivelmente sendo os hospedeiros intermediários, responsáveis pela contaminação de humanos (6,7). O vírus espalhou-se rapidamente internacionalmente (8) causando uma pandemia que acomete milhares de pessoas.

Considerando a origem animal do vírus, é provável que sua relevância não se dê somente em infecções em humanos e possa evoluir para uma enfermidade de caráter zoonótico. Com essa possibilidade, estudos buscam a comprovação da presença do agente em diversas espécies de animais domésticos e quais as similaridades entre essas e o ser humano (8).

O presente estudo visou realizar uma revisão de literatura de diversas publicações científicas abrangendo a possibilidade de os animais serem reservatórios do agente do novo coronavírus, bem como, acentuar a importância da saúde única no controle de doenças através da pesquisa de publicações realizadas até 10 de Junho de 2020.

\section{DESENVOLVIMENTO}

Conforme a Organização Mundial da Saúde Animal (9), foram identificados, através de testes moleculares, animais domésticos positivos para COVID-19 que conviviam com tutores doentes.

Um gato da Bélgica que apresentou sintomatologia gastrointestinal e respiratória foi testado através do exame de reação em cadeia da polimerase em tempo real (RT-PCR), que permite identificar a presença do agente infeccioso no material genético do paciente, o qual demonstrou ser positivo para o novo coronavírus. Ainda, uma tigresa do Zoológico do Bronx em Nova Iorque, EUA, demonstrou sinais clínicos respiratórios também tendo seu diagnóstico confirmado para COVID-19. Mais seis felinos no mesmo zoológico apresentaram manifestações clínicas semelhantes, no entanto, estes animais não foram testados. Acredita-se que os animais foram contaminados por um funcionário assintomático (10).

De acordo com O'Connor et al. (11), gatos infectados por SARS-COV e SARS-COV2 são capazes de desenvolver resposta humoral contra os vírus, corroborando com o estudo de Zhang e colaboradores (12) no qual coletaram amostras de 102 gatos em Wuhan, China, e realizaram o teste de ELISA indireto para titulação de anticorpos. Do total de análises, apenas 15 foram positivas e não houve reação cruzada com o coronavírus causador da peritonite infecciosa felina. Este resultado revela que os gatos são capazes de serem infectados com coronavírus e desenvolver resposta imunológica, contudo, muitas vezes podem ser assintomáticos.

Outros estudos indicam que felinos possam ser hospedeiros acidentais de COVID-19, pois estes possuem somente dois aminoácidos (aa) diferentes de humanos no receptor para o novo vírus. Assim como SARS-COV, SARS-COV-2 invade o organismo pela ligação com o receptor da enzima conversora de angiotensina II (ECA2), a qual é expressa por pneumócitos tipo II no epitélio pulmonar, endotélio vascular, rins e músculo liso $(8,13)$.

No que se refere a cães infectados, no mês de Março de 2020, um macho da raça Pastor Alemão em Hong Kong foi testado positivo, porém no mesmo ambiente havia outro cão sem raça definida que apresentou teste negativo, ambos os animais não apresentaram sinais clínicos. Em Junho, outro cão da raça Pastor Alemão foi o primeiro positivo para COVID-19 nos EUA, confirmado pelo Laboratório Nacional de Serviços Veterinários

Freitas K, Silveira R, Barbosa A. Saúde Única e COVID-19: Revisão sobre o potencial dos animais como reservatórios do vírus. Vet. e Zootec. 2020; 27: 001-007. 
(NVSL) do Departamento de Agricultura dos Estados Unidos (USDA), após manifestação clínica (14-16). Ao contrário dos felinos, cães apresentam cinco aa distintos de humanos no receptor ECA2 (17).

Em outra pesquisa com animais de estimação, Temmam et al. (18) testaram 9 gatos e 12 cães para SARS-COV-2, de estudantes de medicina veterinária, positivos ou com sintomatologia clínica compatível a COVID-19. Nenhum animal positivou no teste de RTPCR e sorologia, embora tenha sido observado sinais clínicos respiratórios e digestivos em alguns destes pacientes. Este estudo demonstra que em situações de exposição natural ao vírus, a suscetibilidade parece ser baixa, ao contrário do apresentado por Shi et al. (8) que descreve que em circunstâncias experimentais, gatos apresentam maior predisposição à infecção.

Shen e colaboradores (19) também investigaram a suscetibilidade de cães, gatos, bovinos e chimpanzés frente ao COVID-19 através da capacidade de utilização do receptor ECA2 de diferentes espécies. Como resultados, identificaram que cães e gatos são mais predispostos à infecção por SARS-COV-2 e que são passíveis de contaminar outros animais não-infectados que convivem com eles.

De qualquer forma, deve-se lembrar que os pêlos dos animais podem ser fontes de reservatório de COVID-19, sendo um fômite propagador do vírus pelo contato usual entre os animais e seus tutores (20). Logo, medidas higiênicas devem ser tomadas após passeios ou contato com possíveis fontes de contaminação. Ressalta-se o alerta para não disseminação de informações falsas sobre a possível contaminação dos animais levando ao abandono desses, sem razão.

Até o momento, não foram detectados animais de produção positivos para o novo coronavírus, sugerindo que estes não são suscetíveis a esta infecção. Entretanto, também pode ser consequência da pouca testagem nesses animais e o menor contato com humanos quando comparados a animais de estimação. Ainda que em surtos passados com outros coronavírus de caráter zoonótico, como SARS-COV e MERS-COV, foram identificados suínos positivos para o primeiro vírus, enquanto ovinos, caprinos e bovinos positivaram para o segundo $(8,11,21-23)$.

Produtos de origem animal também são capazes de atuarem como fômites, como foi detectado pela Organização Mundial da Saúde (24) durante a epidemia de SARS-COV, onde identificaram que o vírus sobrevive na pele suína por mais de 24 horas em temperatura ambiente, porém não se descarta a possibilidade dos demais produtos, como leite e carne, servirem como vetores mecânicos de SARS-COV-2.

Ademais, apesar de não se ter o conhecimento da dose mínima possível de contaminação, já foi detectado a presença do novo coronavírus nas fezes e essa pode ser uma fonte expressiva de contágio principalmente para humanos, que não têm acesso a saneamento básico. Além disso ainda podem contaminar animais que por ventura estejam no ambiente assim como acontece em outras zoonoses com rota de transmissão fecal-oral, tal qual para SARS-CoV e MERS-CoV (25). Estudos demonstram que mesmo após a liberação do vírus das vias respiratórias, sua liberação nas fezes pode continuar ativa (26), sendo uma importante via de propagação em locais com precário tratamento do esgoto ou lixo.

\section{CONCLUSÃO}

Dessa forma, é notório o potencial zoonótico do novo coronavírus a partir de estudos já existentes como os revisados, porém para se afirmar isso são necessários mais estudos ainda sobre o tema. Portanto, evidencia-se a importância da ação da saúde única de forma multiprofissional, na qual atuam médicos, enfermeiros, médicos veterinários, entre tantos, diante de todas fontes envolvidas na disseminação de doenças, como o COVID-19. 


\section{REFERÊNCIAS}

1. Chen N, Zhou M, Dong X, Qu J, Gong F, Han Y. Epidemiological and clinical characteristics of 99 cases of 2019 novel coronavirus pneumonia in Wuhan, China: a descriptive study. Lancet. 2020;395(10223):P507-13. doi: https://doi.org/10.1016/S01406736(20)30211-7.

2. Decaro N, Martella V, Saif LJ, Buonavoglia C. COVID-19 from veterinary medicine and one health perspectives: what animal coronaviruses have taught us. Res Vet Sci. 2020;131:213. doi: 10.1016/j.rvsc.2020.04.009.

3. Buonavoglia C, Decaro N, Martella V, Elia G, Campolo M, Desario C, et al. Canine coronavirus highly pathogenic for dogs. Emerg Infect Dis. 2006;12(3):492-4. doi: 10.3201/eid1203.050839.

4. Salim Mattar V, Marco González T. Zoonotic emergence of coronavirus: a potential public risk for Latin America. Rev MVZ Cordoba. 2018;23(3):6775-77. doi: http://dx.doi.org/10.21897/rmvz.1408.

5. Rodriguez-Morales AJ, Cardona-Ospina JA, Gutiérrez-Ocampo E, Villamizar-Peña R, Holguin-Rivera Y, Escalera-Antezana JP, et al. Clinical, laboratory and imaging features of COVID-19: a systematic review and meta-analysis. Travel Med Infect Dis. 2020;34:101623. doi: 10.1016/j.tmaid.2020.101623.

6. Lam TT, Jia N, Zhang YW, Shum MH, Jiang JF, Zhu HC, et al. Identifying SARS-CoV-2related coronaviruses in Malayan pangolins. Nature. 2020;583(7815):282-5. doi: 10.1038/s41586-020-2169-0.

7. Wong MC, Javornik Cregeen SJ, Ajami NJ, Petrosino JF. Evidence of recombination in coronaviruses implicating pangolin origins of nCoV-2019. bioRxiv. Forthcoming 2020. doi: 10.1101/2020.02.07.939207.

8. Shi J, Wen Z, Zhong G, Yang H, Wang C, Huang B, et al. Susceptibility of ferrets, cats, dogs, and other domesticated animals to SARS-coronavirus 2. Science. 2020;368(6494):101620. doi: 10.1126/science.abb7015.

9. World Organisation for Animal Health - OIE. Information received on 07/04/2020 from Dr Thomas Sit, Chief Veterinary Officer / Assistant Director (Inspection \& Quarantine), Agriculture, Fisheries and Conservation Department, Hong Kong Special Administrative Region Government, Hong Kong, Hong Kong [Internet]. Paris: OIE; 2020 [cited 2020 Jun 5]. (Follow-up report No. 1 (Final report)). Available from: https://www.oie.int/wahis_2/public/wahid.php/Reviewreport/Review?page_refer=MapFullEv entReport\&reportid=33892

10. United States Department of Agriculture - USDA. USDA statement on the confirmation of COVID-19 in a tiger in New York [Internet]. Washington: USDA; 2020 [cited 2020 Jun 5]. Available from: https://www.aphis.usda.gov/aphis/newsroom/news/sa_by_date/sa-2020/nyzoo-covid-19 
11. O'Connor AM, Totton SC, Sargeant JM. A rapid review of evidence of infection of pets and livestock with human-associated coronavirus diseases, SARS, MERS, and COVID-19, and evidence of the fomite potential of pets and livestock [Internet]. East Lansing, MI: SYREAF; 2020 [cited 2020 Jun 5]. Available from: http://www.syreaf.org/wpcontent/uploads/2020/04/Rapid-Review-of-pets-as-fomites_3.pdf

12. Zhang Q, Zhang H, Huang K, Yang Y, Hui X, Gao J, et al. SARS-Cov-2 neutralizing serum antibodies in cats: a serological investigation. bioRxiv. Forthcoming 2020. doi: https://doi.org/10.1101/2020.04.01.021196.

13. Lan J, Ge J, Yu J, Shan S, Zhou H, Fan S, et al. Structure of the SARS-CoV-2 spike receptor-binding domain bound to the ACE2 receptor. Nature. 2020;581:215-20. doi: 10.1038/s41586-020-2180-5.

14. Almendros A, Gascoigne E. Can companion animals become infected with Covid-19? Vet Rec. 2020;186(13):419-20. doi: 10.1136/vr.m1322.

15. Bryner J. Cat infected with COVID-19 from owner in Belgium. Live Science [Internet]. 2020 [cited 2020 Jun 5]. Available from: https://www.livescience.com/cat-infected-covid-19from-owner.html

16. United States Department of Agriculture - USDA. Confirmation of COVID-19 in pet dog in New York [Internet]. Washington: USDA; 2020 [cited 2020 Jun 5]. Available from: http://aphis.usda.gov/aphisnewsroom/stakeholder-info/sa_by_date/sa-2020/sa-06/sars-cov-2$\operatorname{dog}$

17. Liu Z, Xiao X, Wei X, Li J, Yang J, Tan H, et al. Composition and divergence of coronavirus spike proteins and host ACE2 receptors predict potential intermediate hosts of SARS-CoV-2. J Med Virol. 2020;92(6):595-601. doi: 10.1002/jmv.25726.

18. Temmam S, Barbarino A, Maso D, Behillil S, Enouf V. Absence of SARS-CoV-2 infection in cats and dogs in close contact with a cluster of COVID-19 patients in a veterinary campus. bioRxiv. Forthcoming 2020.

19. Shen M, Liu C, Xu R, Ruan Z, Zhao S, Zhang H, et al. SARS-CoV-2 Infection of Cats and Dogs? Preprints. 2020:2020040116.

20. Ristow LE, Carvalho OV, Gebara RR. COVID-19 em felinos, seu papel na saúde humana e possíveis implicações para os seus tutores e para a vigilância em saúde. Epidemiol Serv Saude. 2020;29(2):e2020228. doi: 10.5123/s1679-49742020000300005.

21. Wang M, Jing HQ, Xu HF, Jiang XG, Kan B, Liu QY, et al. [Surveillance on severe acute respiratory syndrome associated coronavirus in animals at a live animal market of Guangzhou in 2004]. Zhonghua Liu Xing Bing Xue Za Zhi. 2005;26(2):84-7. PMID: 15921605. Chinese.

22. Kandeil A, Gomaa M, Shehata M, El-Taweel A, Kayed AE, Abiadh A, et al. Middle East respiratory syndrome coronavirus infection in non-camelid domestic mammals. Emerg Microbes Infect. 2019;8(1):103-8. doi: 10.1080/22221751.2018.1560235.

23. Deng J, Jin Y, Liu Y, Sun J, Hao L, Bai J, et al. Serological survey of SARS-CoV-2 for experimental, domestic, companion and wild animals excludes intermediate hosts of 35 
different species of animals. Transbound Emerg Dis. 2020;67(4):1745-9. doi: $10.1111 /$ tbed.13577.

24. World Health Organization - WHO. First data on stability and resistance of SARS coronavirus compiled by members of WHO laboratory network [Internet]. Geneva: WHO; 2003 [cited 2020 Jun 8]. Available from: https://www.who.int/csr/sars/survival_2003_05_04/en/

25. Yeo C, Kaushal S, Yeo D. Enteric involvement of coronaviruses: is faecal-oral transmission of SARS-CoV-2 possible? Lancet Gastroenterol Hepatol. 2020;5(4):335-7. doi: 10.1016/S2468-1253(20)30048-0.

26. Wu Y, Guo C, Tang L, Hong Z, Zhou J, Dong X, et al. Prolonged presence of SARSCoV-2 viral RNA in faecal samples. Lancet Gastroenterol Hepatol. 2020;5(5):434-5. doi: $10.1016 / \mathrm{S} 2468-1253(20) 30083-2$.

Recebido em: 29/06/2020

Aceito em: 24/08/2020 